\title{
NASALANCE IN UTTERANCES OF HEARING-IMPAIRED SPEAKERS
}

\author{
SAMUEL G. FLETCHER \\ Department of Biocommunication, University of Alabama in Birmingham, Birmingham, Alabama \\ 35294 \\ DAVID A. DALY \\ University of Michigan
}

\begin{abstract}
Instrumental comparisons of sound from the nose and mouth expressed in percent nasalance, articulation errors, and speech rate are used in this study to contrast utterances of 50 speakers with severe hearing impairment and 64 with normal hearing. Results of the study revealed that $54 \%$ of the hearing-impaired subjects had nasalance ratio scores greater than two standard deviations above the mean of the group with normal auditory acuity. No general relationship was found between the number or type of articulation errors and the nasalance scores. Rate of speaking was significantly related to the nasalance scores of the normal group but not those of the hearing-impaired group. Tonagrams displaying the variations in nasalance revealed small, 1 to $5 \%$ spike-shaped fiuctuations in the displays of the utterances from both groups. Additional prolonged bursts of nasalance in excess of a $30 \%$ change in the ratio between the nasal and oral signals were common in the displays from the hearing-impaired group but not in those of the group with normal acuity. Possible sources and perceptual effects of these phenomena are discussed.
\end{abstract}

\section{Introduction}

The purpose of this investigation was to compare nasalance measurements of sound from the nose and from the mouth during utterances for speakers with hearing impairments and speakers with normal auditory acuity. A second purpose was to examine interrelationships between nasalance, rate of speech production, and errors in speech articulation among the hearing impaired.

\section{Method}

\section{Subjects}

The experimental subjects were 27 females and 23 males from the Alabama School for the Deaf in Talladega, Alabama. They ranged from 7 through 21 years of age with a mean age of 15.9. The mean hearing loss in the better ear was $83 \mathrm{~dB}$ HTL (re 1964 ISO standards). None had other known physical impairments that would interfere with speech.

The normal control subjects consisted of 32 females and 32 males with no clinically significant speech defects and who ranged in age from 7 to 25 years with 
a mean of 13.5. All had passed a pure tone hearing screening test of $250-4,000 \mathrm{~Hz}$ at $15 \mathrm{~dB}$ HTL.

\section{Instrumentation}

The instrument used to collect data was a Quan-Tech TONAR II system. The instrument uses a baffle against the upper lip and between two microphone chambers to maintain the separation of the oral and nasal sounds as they emerge from the respective oral and nasal cavities. The sound pressure variations are converted by microphones into corresponding nasal and oral electrical signals. These signals are filtered by tunable bandpass filters usually set at a common center frequency of $500 \mathrm{~Hz}$ with a 3-dB band width of $300 \mathrm{~Hz}$. Each filtered signal is rectified and the results averaged using an $\mathrm{RC}$ network having selectable time constants of $0.1,1$, or $10 \mathrm{sec}$. The output values from the averaging networks in the nasal and oral channels denoted by $\overline{\mathrm{N}}$ and $\overline{\mathrm{O}}$ are used to compute a nasalance ratio defined as:

$$
\text { Nasalance }=\overline{\mathrm{N}} / \overline{\mathrm{N}}+\overline{\mathrm{O}} \times 100 .
$$

Because the ratio $R$ lies between 0 and 1 , it is conveniently multiplied by 100 and expressed as percent nasalance. The resulting computation is indicated on a digital counter on the instrument control panel. It may also be displayed in analog form using an $\mathrm{X}-\mathrm{Y}$ recorder.

The instrument will accept either live voice directly from the microphones in the sound separator assembly against which the speaker's face is placed or from a dual channel tape recording of such material. In the 0.1 -sec time constant (T.C.) mode of operation, the nasalance value reflects the moment-by-moment variations in nasal versus oral resonance during vocalization and drops to zero during pauses in speech. In the 10-sec T.C. mode the measurements reflect the more general relationship of the signals. And since the filters discharge slowly and at the same rate, the calculated values are maintained during pauses in vocalization. Thus, pauses such as found frequently in utterances of persons with severe hearing impairment have little effect on the nasalance values generated.

\section{Stimulus Material}

Three sets of stimulus materials were used: Fletcher's (1972) "Zoo Passage," a sustained utterance of an isolated $\mid a /$ vowel and the Goldman-Fristoe Speech Articulation Test. The Zoo Passage was written on a 2 nd to 3 rd grade reading level. It contains no nasal consonants; otherwise its phonemic distribution corresponds to that of spoken English. 


\section{Data Collection Procedures}

The data from the experimental subjects were collected at the Alabama School for the Deaf. To record the speech, the output of TONAR II was coupled to a TEAC Model 610 audio tape recorder. Two examiners and one educational aid were present. One examiner positioned the subjects against the sound separator of TONAR II and monitored this position closely during data collection to be sure that adequate sound separation was retained during speaking efforts. If the person had difficulty in reading, this examiner also helped him/her repeat the Zoo Passage into the sound separator. Tape recording was stopped during periods of instruction. The educational aid, who was trained in sign language, helped explain the procedural requirements to the subjects and assisted when necessary in eliciting responses. The other examiner operated the instruments and monitored the incoming data as it was tape recorded. A $500-\mathrm{Hz}$ calibration tone was placed on each channel of the recorder preceding the responses of each subject. These tones were used in later analysis to reestablish the interchannel intensity balance so that the ratios would be recalculated accurately.

The articulation testing was conducted by a second pair of examiners in an adjoining room. Standard procedures were followed in obtaining and scoring the responses from live voice.

Data from the control group were already available from a normative study of individuals examined in the Biocummunication Laboratory at the University of Alabama in Birmingham (UAB). The material was again read out loud to some of the younger children in this group, who then repeated the sentences into the sound separator of TONAR II to be recorded. Prior experience had shown no differences in the nasalance ratio whether the material was read or repeated into the instrument.

\section{Analysis Method}

Analyses of the tape recorded responses were conducted in the Biocommunication Laboratory of the University of Alabama in Birmingham. The output of the tape recorder was coupled to a Houston "Omnigraphic'' Model $2111 \mathrm{X}-\mathrm{Y}$ recorder, and the calibration tones were played through TONAR II set at a $300-\mathrm{Hz}$ band width around a central frequency of $500 \mathrm{~Hz}$. The balance control between the two channels of the recorder was adjusted to obtain equal intensity of the two tones, as indicated by a $50 \%$ nasalance reading on TONAR II and a $500-\mathrm{mV}$ $\mathrm{Y}$-scale reading on the $\mathrm{X}-\mathrm{Y}$ recorder. The time base of the $\mathrm{X}-\mathrm{Y}$ recorder was set at $5 \mathrm{sec} / \mathrm{inch}$, and the $\mathrm{Y}$-sensitivity was set so that a $2 \%$ change in the nasalance ratio from TONAR II would cause the recording pen to be raised one small scale division of 0.1 inch on the chart paper. These settings permitted resulting tonagrams to be read in percent nasalance to the nearest $1 \%$ value. 
Two overlaid tracings were charted on a tonagram for each of the two utterances, one with the time averaging constant set at $0.1 \mathrm{sec}$ and one with it set at 10 sec. Three scores were obtained from each tonagram of the Zoo Passage: the minimum and maximum values from the $0.1-\mathrm{sec}$ tracing and the average value from the 10-sec averaged tracings. To derive a more accurate estimate of the extremes, the two low points and the two high points on the $0.1-\mathrm{sec}$ T.C. curve were each combined and averaged for the minima and maxima (low and high end of the range), respectively.

Variability in nasalance of the $/ a$ / vowel by subjects in the normative group was generally less than $2 \%$; therefore, only the $10-\mathrm{sec}$ averaged score was calculated for this utterance.

The time required to utter the 75-word Zoo Passage was obtained from the time base of the tonagrams. The subjects were specifically instructed to speak all words in the passage. Transformations made later to compare the results in terms of speech rate thus contain a slight overestimate of the actual speech rate of some of the hearing-impaired subjects who omitted words despite prior admonition to say all of them.

The data were statistically analyzed under the direction of Dr. David Hurst, Professor and Chairman of the Department of Biostatistics, UAB. The analyses made use of appropriate parts of the Statistical Analysis System (SAS) by Barr and Goodnight (1972).

\section{Results}

The means, standard deviations, and statistical comparisons of the nasalance data are displayed in Table 1. These results show that as a group the hearingimpaired speakers allowed a much greater proportion of the sound to escape through the nose than did those in the normative sample regardless of the material spoken. The among-speaker variation was also much greater in the hearingimpaired group. All of the differences in nasalance shown between the two groups were statistically significant $(p<0.001)$.

A sequential sum-of-squares analysis of variance was used to examine possible interactions among nasalance, age, sex, and normal or hearing-impaired speaker classification. In this part of the data analysis the only major factor that emerged consistently as statistically different between the two groups was that the hearingimpaired group had greater nasalance. A modest interaction $(p<0.05)$ was found between sex and the deaf versus normal hearing category on 10 -sec averaged scores of the Zoo Passage. The female hearing-impaired subjects in this particular sample obtained somewhat lower nasalance scores than the males $(19.4 \%$ vs. $24.0 \%$ ) and the normal females obtained slightly higher nasalance scores than the normal males $(9.1 \%$ vs. $7.8 \%)$.

To clarify the distribution of the nasalance scores somewhat further, the data 
TABLE 1

Comparison of Relative Acoustic Energy in Sound from the Oral Cavity and the Nasal Cavities during the Utterances by Hearing-Impaired and Normal Speakers (All Measurements Are Fxpressed in Percent Nasalance)

\begin{tabular}{|c|c|c|c|c|c|c|c|}
\hline \multirow[b]{2}{*}{ Utterance } & \multirow[b]{2}{*}{ Measurement } & \multicolumn{2}{|c|}{$\begin{array}{l}\text { Hearing-impaired } \\
\text { group }(N=50)\end{array}$} & \multicolumn{2}{|c|}{$\begin{array}{l}\text { Normative group } \\
(N=64)\end{array}$} & \multirow[b]{2}{*}{$t$} & \multirow[b]{2}{*}{$P$} \\
\hline & & Mean & $\mathrm{SD}$ & Mean & SD & & \\
\hline \multirow[t]{3}{*}{$|a|$} & $\begin{array}{l}\text { Low end of } \\
\text { range }\end{array}$ & 12 & 10.86 & - & - & - & - \\
\hline & $\begin{array}{l}\text { High end of } \\
\text { range }\end{array}$ & 28 & 14.45 & - & - & - & - \\
\hline & $\begin{array}{l}\text { 10-sec } \\
\text { average }\end{array}$ & 19 & 12.32 & 6 & 6.88 & 5.77 & 0.0001 \\
\hline \multirow[t]{3}{*}{ Zoo Passage } & $\begin{array}{l}\text { Low end of } \\
\text { range }\end{array}$ & 6 & 5.89 & 3 & 1.76 & 3.46 & 0.001 \\
\hline & $\begin{array}{l}\text { High end of } \\
\text { range }\end{array}$ & 46 & 18.67 & 18 & 5.37 & 10.68 & 0.0001 \\
\hline & $\begin{array}{l}\text { 10-sec } \\
\text { average }\end{array}$ & 22 & 12.55 & 9 & 3.68 & 7.34 & 0.0001 \\
\hline
\end{tabular}

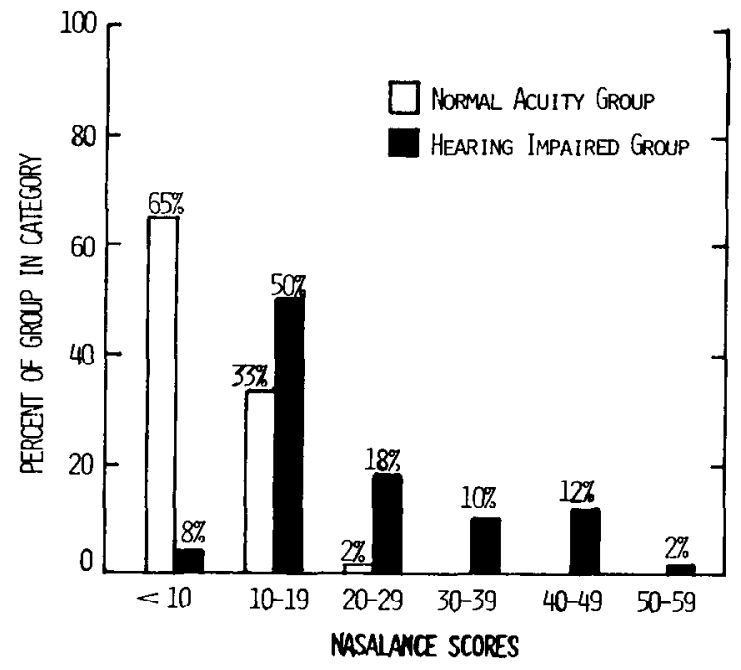

Fig. 1. Accumulative distribution of scores by subjects classed as having normal auditory acuity (open bars) or hearing impairment (solid bars) within six levels of nasalance.

were plotted in a histogram, shown in Fig. 1. The plot reveals substantial overlap between the two groups of subjects in the normal or near-normal range of $10 \%$ to $19 \%$ nasalance; however, $72 \%$ of the hearing-impaired subjects achieved nasalance scores on the Zoo Passage utterance that were more than one standard 
deviation higher than the mean of the control group. Fifty-four percent of the scores were more than two standard deviations above the mean of the control group. A wide distribution of nasalance scores in the hearing-impaired group is clearly evident.

The speaking times of the hearing-impaired speakers to utter the 75-word Zoo Passage ranged from 26 to $105 \mathrm{sec}$ with a mean of 47 and a standard deviation of $18.3 \mathrm{sec}$. The corresponding time required for the subjects with normal hearing acuity to speak the paragraph ranged from 19 to $34 \mathrm{sec}$ with a mean of 26 and a standard deviation of $4.9 \mathrm{sec}$. Thus, as a group the hearing-impaired speakers spoke much more slowly than their normal hearing peers. The mean speaking times would be roughly equivalent to $95 \mathrm{wpm}$ for the hearing-impaired group and $173 \mathrm{wpm}$ for the control group. It should be noted that some of the hearingimpaired speakers omitted words. For this reason the $95-w p m$ rate calculated for this group is somewhat inflated.

Correlation coefficients were computed to examine the relationship between the time required to utter the Zoo Passage and the nasalance scores. The resulting correlations were 0.13 for the speakers in the hearing-impaired group and 0.30 for those in the control group. Only that for the control group was found to be statistically significant $(p<.01)$.

Speech articulation errors were common among the hearing-impaired speakers: of 63 possible errors on the Goldman-Fristoe Test the mean number of errors was 26. The most common articulatory errors were of the $/ \mathrm{s} / \mathrm{blends} / \mathrm{s} 1 /, / \mathrm{st} /$, and $/ \mathrm{skw} /$ and the sibilants $/ \mathrm{s} /, / \mathrm{z} /, / \int /,|z h /|, t \int /$, and $/ \mathrm{d} z h /$. Over half of these sounds were produced in error by the speakers in the hearing-impaired group.

Among the hearing-impaired, interrelationships were found to be low between the measurements of nasalance and the articulatory errors. No significant correlations were found between any class of articulation error and nasalance scores in the connected speech utterances of the Zoo Passage or between the utterances of the isolated $\mid a /$ vowel and four of the eight articulatory sound classes: sibilants, plosive blends, nasals, and fricative blends. Low but significant $(p<0.05)$ negative correlations ranging from -0.28 to -0.32 were found between nasalance in the utterances of the isolated /a/ vowel and /s/ blend, liquid, plosive, and nonsibilant fricative sound classes.

The relation between articulation scores and speaking time of the Zoo Paragraph was also examined in the hearing-impaired speaker responses. The correlation coefficient was 0.56 , indicating a moderate level of correspondence between time required to utter the passage and the general accuracy of speech production in these individuals.

Closer examination of the configuration of the nasalance curves revealed an additional important characteristic of the pattern that was obscured when only averaged scores were considered. In the $0.1-\mathrm{sec}$ T.C. displays of nasalance from utterances of both the normal and the hearing-impaired groups the proportion of 
the phonic stream passing through the oral and nasal cavities varied continuously throughout each utterance. This is exemplified by the small, spike-shaped fluctuations evident in each of the $0.1-\mathrm{sec}$ T.C. curves of the tonagrams of the normal speaker shown in Fig. 2 and of the hearing-impaired speakers shown in

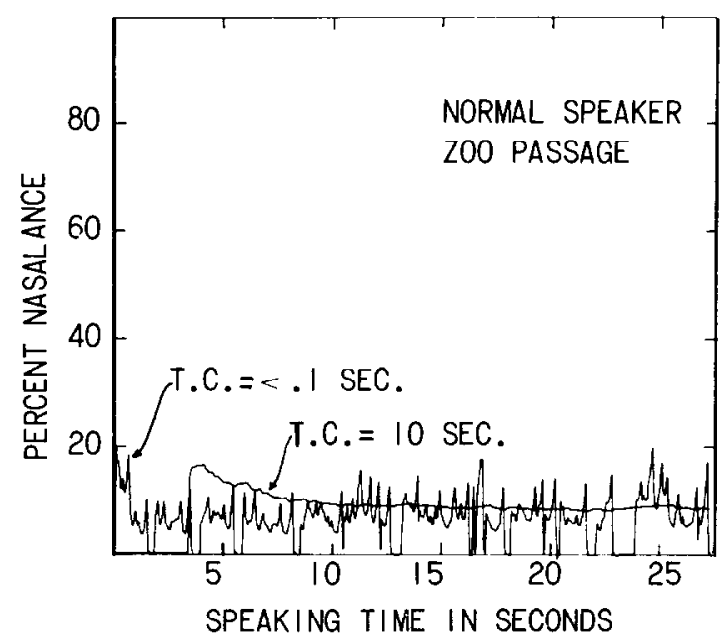

Fig. 2. Tonagram of a normal speaker during utterance of the Zoo Passage. Note that although momentary spikes are shown in the nasalance fluctuations, the type of prolonged bursts evident in the tonagrams of the hearing-impaired speakers was not present in this speaker's utterances.

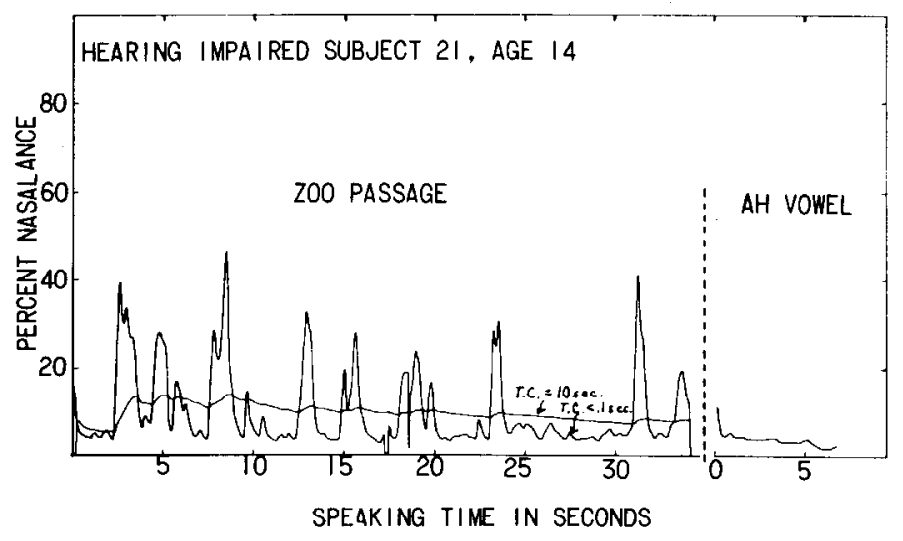

Fig. 3. Tonagram demonstrating "bursts" as well as small fluctuations or "spikes" of nasalance in the utterances of a hearing-impaired speaker during production of the Zoo Passage. The smaller fluctuations are typical of all utterances of all connected speech utterances. Bursts are normally not found in utterances such as this which contain no nasal consonants. In this instance they are felt to represent momentary inadequacies in palatopharyngeal valving. 


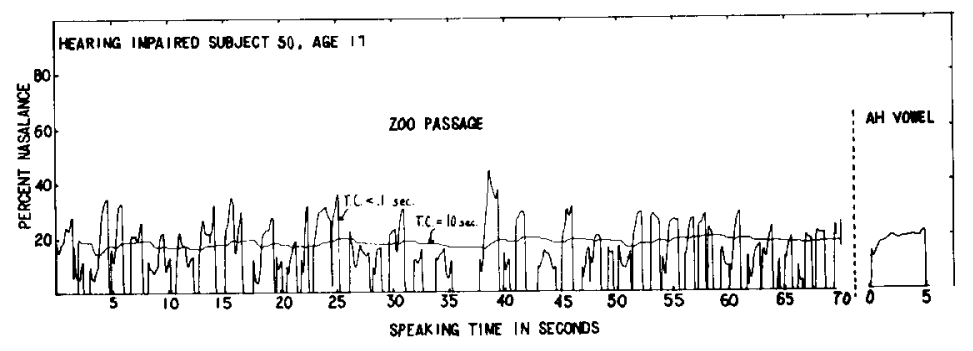

Fig. 4. Tonagram demonstating supranasalance against a background of very slow speech production (64-wpm rate). Frequent bursts of nasal sound are evident, which appear to be the major influence causing a mild elevation in the 10-sec averaged curve.

Figs. 3 and 4 . The presence of these small fluctuations with a magnitude of 1 to $5 \%$ nasalance likely reflects small fluctuations in vocal energy and in the transmission characteristics of the vocal tract.

In contrast to the spikes of nasalance, the configuration of the 0.1-sec T.C. curves from the utterances of the hearing-impaired speakers often also displayed rather prolonged bursts of nasal resonance with a change of greater than $30 \%$ in the nasalance ratio. Such bursts were rarely observed in the tonagrams of the speakers with normal acuity. The appearance of the nasalance bursts is particularly well demonstrated in the display of the utterance shown in Fig. 3. In sharp contrast with an averaged nasalance level of $8 \%$ to $10 \%$, bursts are seen that exceeded a $40 \%$ change in the nasalance ratio. Similar bursts are also displayed in the tonagram of the utterance in Fig. 4 from a different hearing-impaired speaker. In the latter utterance the bursts were frequent and appeared to be the main source of the elevation of the averaged score to a level of $20 \%$, which is outside the normal range.

\section{Discussion}

As noted earlier, nasalance is a term coined to represent a physical relationship between nasal and oral sound. The term nasality is reserved to designate the perceptual impression of nasal quality. Because perceived nasality is difficult to scale, as well-shown in the study by Bradford, Brooks, and Shelton (1964) and since perceptual ratings have been the primary means of establishing the existence of excessive nasal resonance, it is not surprising to find considerable variation in reports from studies investigating nasal voice quality in hearing-impaired speakers.

An early study of speech of the deaf by Hudgins and Numbers (1942) suggested nasality to be rather rare in this population. They used two listeners to judge the 
recorded vocal responses of 192 deaf children during utterances of 10 sentences. Among the results of their ratings were the number of articulation errors involving the nasal consonants and the number of words with excessive nasality. Of the 19,470 possible errors in consonant categories only 408 , or $2.1 \%$, involved nasal consonants and of 15,140 possible errors in vowel categories only 129 , or $0.8 \%$, were judged to be overly nasalized.

In 1955 Penn studied the voice and speech patterns of 671 veterans with conductive hearing losses and 1,086 with sensori-neural losses. A single examiner was used to elicit and judge speech responses. One of eight classifications used to identify deviations in vocal quality was excessive nasal resonance. Of the veterans with sensori-neural losses $43 \%$ were judged to have excessively nasal voices. Of those with conductive losses $25 \%$ were so classified.

In a more recent study, Colton and Cooker (1968) used 30 listeners to judge nasality in the recorded utterances of two sentences from a six-sentence passage spoken by 28 young adult deaf speakers. All but two of these speakers were judged to be more than moderately nasal on the introductory sentence of the paragraph, and all speakers were so judged on the second sentence. Thus, in the three studies, which have sought to document nasality in heaving-impaired speakers, one concluded it is very rare, one that somewhat less than half are overly nasal, and one that virtually all such speakers have excessive nasality.

In the present study nasalance was significantly higher in the hearing-impaired speakers than in the normal control group. Only $28 \%$ of the hearing-impaired subjects achieved nasalance scores within onc standard deviation of the mean control group while the scores of over half of them (54\%) were two standard deviations above the mean level of the control group.

McClumpha (1966) compared palatopharyngeal valving patterns through cinefluorographic observations of five deaf speakers and five speakers with normal hearing acuity during the repeated utterances of the syllables $/ \mathrm{pi} /, / \mathrm{l} /$, and $/ \mathrm{pu} /$. All normal speakers were found to achieve and maintain contact of the palate with the pharyngeal wall throughout production sets of seven syllables each. Only one of the hearing-impaired speakers followed this pattern. Of the four remaining hearing-impaired speakers one never achieved closure. Despite the fact that no nasal consonants were present, the other three hearing-impaired speakers demonstrated variable closure patterns during repetition of these syllables. McClumpha noted that the contact between the palate and the pharyngeal wall was typically obtained during production of the consonant portion of the utterances but often not during the vowels. Lack of palatopharyngeal closure during vowel production could well be the major source of the nasalance bursts observed in the present study. Documentation of this possibility would require simultaneous radiographic and acoustic observations.

Colton and Cooker (1968) speculated that the excessive nasality judged to be present in the utterances of the deaf speakers they studied may have been the 
consequence of reduced speaking tempo rather than abnormal palatopharyngeal function. This hypothesis arose from the finding that when seven individuals with normal speech spoke at a slow, one-word-at-a-time tempo, they were judged to be more nasal than when they spoke at their normal rate. Furthermore, five of the seven word-by-word samples were judged to be as nasal as many of the samples spoken by a group of deaf speakers. This interpretation was supported by observations of Bzoch (1965) who found discontinuities in palatopharyngeal contact as a normal subject reduced his speaking rate. Bjork (1961) also reported momentary interruptions in palatal contact with the pharyngeal wall during pauses in speech by normal speakers.

The correlation between the time required to read the Zoo Passage and the nasalnance scores of the hearing-impaired speakers was very low and statistically nonsignificant. We suggest that the elevated nasalance in this group likely reflected the inability of such speakers to monitor their speech output accurately and thus achieve the fine control of palatopharyngeal valving typical of speakers with normal auditory acuity.

Some possible perceptual effects of the signals represented by the nasalance bursts of the hearing-impaired speaker utterances may be hypothesized. Powers (1973) indicated that the control system of any perceptual processing must contain a filtering effect that allows an observer to average incoming signals and remove disturbances that come and go rapidly. This is necessary to permit emergence and identification of the main elements in the perceptual field. We suggest that the rapid fluctuations of nasal resonance denoted by the spikes in the nasalance displays could in like manner be filtered from the perceptual field by a speaker with normal hearing. If so, their presence would be undetected. Conversely, when slower, more prominent bursts occurred, especially those irregular in their time sequence, they would be more likely to be detected and processed as possible phonetic elements in the language spoken.

Fry (1963) suggested that in speech recognition the phonemic elements are represented at the acoustic level by a system of relations rather than by a set of absolute values. He noted further that identification of phonetic units from the acoustic input seems to depend largely on weighing several cues and deciding upon the evidence what the unit is.

In other data from speakers with normal hearing acuity one of the present writers (S.G.F.) observed that nasal consonants are expressed in tonagrams as bursts in nasal ance that typically exceed a change of $30 \%$ in the output ratio. Interestingly, the graphical representation of these bursts resemble rather closely those displayed in the tonagrams from the hearing-impaired speakers in their utterance of the Zoo Passage. The Zoo Passage, however, contains no nasal consonants. Thus, it seems likely that perceptual confusion could arise from the presence of nasal consonant-like acoustic cues in a context where such consonants were not actually present. At a perceptual level this could lead to a mismatch between detection of 
the significant changes in nasal resonance and the phonetic identification of the sounds spoken. That is, the nasalance bursts could well be misclassified as nasal consonants. At the very least the conflicting information from such acoustical aberrations and the context of the spoken message would seem likely to place an unusual burden upon a listener. It would require considerable adeptness in use of the listener's knowledge concerning the constraints of the language spoken to resolve the uncertainties and correct the apparent errors in the acoustic output. This could be an important reason why speech of the hearing-impaired person is so difficult to understand by many listeners. This possible relationship warrants considerable further attention.

Appreciation is expressed to Mrs. Zackie Bosarge, Mr. Charles Johnson, Principal, and others at the Alabama School for the Deaf, Talladega. This study was sponsored by the Regional Rehabilitation Research and Training Center Grant 16-P-5680 from the U.S. Social Rehabilitation Services and Project 910, U. S. Maternal and Child Health Service, HEW.

\section{References}

Barr, A. J., Goodnight, J. H. Statistical analysis system. Raleigh: North Carolina State University Press, 1972.

Bjork, L. Velopharyngeal function in connected speech. Acta Radiol. [Diag.] [Suppl.] Stockh.), 1961, 202.

Bradford, L. J., Brooks, A. R., Shelton, R. L., Jr. Clinical judgements of hypernasality in cleft palate children. Cleft Palate J., 1964, 1, 329.

Bzoch, K. R. Variations in velopharyngeal valving as a function of syllabic changes in repeated CV syllables. Paper presented at ASHA Annual Convention, Chicago, 1965.

Colton, R. H., Cooker, H. S. Perceived nasality in the speech of the deaf. J. Speech Hearing Res., 1968, 11, 553-559.

Fletcher, S. G. Contingencies for bioelectronic modification of nasality. J. Speech Hearing Dis., 1972, 37, 329-346.

Fry, D. B. Theoretical aspects of mechanical speech recognition. In: Engineering summer conference on automatic speech recognition, Vol. 1. Ann Arbor: The University of Michigan, 1963.

Hudgins, C. V., Numbers, F. C. An investigation of the intelligibility of speech of the deaf. Genet. Psychol. Monogr., 1942, 25, 289-392.

McClumpha, S. L. Cineflurographic investigation of velopharyngeal function in selected deaf speakers. Master's thesis, University of Florida, 1966.

Penn, J. P. Voice and speech patterns of the hard of hearing. Acta Otolaryngol. [Suppl.] Stockh.), $1955,124$.

Powers, W. T. Behavior: The control of perception. Chicago: Aldine Publishing Co., 1973. 